\title{
Association between Serum-Ferritin Levels and Sleep Duration, Stress, Depression, and Suicidal Ideation in Older Koreans: Fifth Korea National Health and Nutrition Examination Survey 2010-2012
}

\author{
Kyung Mi Kim ${ }^{1}$, Hye Rim Hwang ${ }^{1,2,}$, Yun Jin $\mathrm{Kim}^{1,2}$, Jeong Gyu Lee ${ }^{1,2}$, Yu Hyeon Yi, ${ }^{1,2}$, Young Jin Tak ${ }^{1,2}$, \\ Seung Hun Lee ${ }^{1,2}$, Sung In Chung ${ }^{1}$ \\ 'Department of Family Medicine, Pusan National University Hospital, Busan, Korea \\ ${ }^{2}$ Department of Family Medicine, Pusan National University School of Medicine, Busan, Korea
}

\begin{abstract}
Background: Serum-ferritin levels may be associated with psychiatric symptoms among the elderly; however, this association has not been extensively studied. The aim of this study was to evaluate the association between serumferritin levels and mental health issues in older Koreans.

Methods: This cross-sectional study included large-scale national data from the Korea National Health and Nutrition Examination Survey 2010-2012. In total, 1,802 older Koreans ( $\geq 65$ years old) were included. The assessed psychiatric symptoms included short sleep duration, stress, depressed mood, and suicidal ideation. Any association between serum-ferritin levels and mental health issues according to sex was examined using logistic regression analysis.

Results: The prevalence of short sleep duration, stress, depressed mood, and suicidal ideation was $50.9 \%, 21.7 \%$, $18.2 \%$, and $22.5 \%$, respectively. In men, suicidal ideation decreased with increased serum-ferritin levels after multivariate adjustment for sociodemographic and lifestyle factors and comorbidities. The odds ratios (OR) and 95\% confidence interval (CI) of suicidal ideation by quartiles of serum-ferritin level were 1 (reference), 1.132 (95\% CI, 0.569-2.252), 0.453 (95\% CI, 0.217-0.945), and 0.608 (95\% CI, 0.295-1.253), respectively, (P for trend=0.039). In women, no trend was observed. However, compared to the lowest quartile, short sleep duration $(<7 \mathrm{~h})$ and stress perception were significantly decreased in the third quartile, with odds ratios of 0.601 (95\% CI, 0.461-0.888), and 0.606 (95\% CI, 0.386-0.952), respectively.

Conclusion: Moderate serum-ferritin levels were associated with decreased risk for suicide ideation in men, and were associated with decreased short sleep duration and stress perception in women.
\end{abstract}

Keywords: Aged; Ferritins; Mental Health; Republic of Korea

Received: July 3, 2018, Revised: September 25, 2018, Accepted: October 12, 2018

*Corresponding Author: Hye Rim Hwang https://orcid.org/0000-0001-7658-3749

Tel: +82-51-240-7000, Fax: +82-51-240-7843, E-mail: hezera83@naver.com 


\section{INTRODUCTION}

Psychological wellbeing is a prominent component of public health, and insufficient sleep affects mental and physical health among the elderly. ${ }^{1)}$ Stress can cause physical problems such as diabetes mellitus, hypertension, and cardiovascular disease, as well as mental problems such as cognitive disorder and depression. Individuals who are often exposed to stress may become depressed and develop suicidal ideation in severe cases. ${ }^{2)}$ Depressive symptoms have a high prevalence rate in Korea; according to the 2011 Korea Health statistics, $4.8 \%$ of adult men, and $10.1 \%$ of adult women experience depressive symptoms. ${ }^{3)}$ Depression may be an important risk factor for suicide, and Korea has a high suicide rate among the Organization for Economic Cooperation and Development countries. ${ }^{4}$

Iron is an essential inorganic substance for normal body physiology. ${ }^{5)}$ It is an important component of hemoglobin and is involved in blood oxygen activity, brain function, cognition, and behavior. It is also essential for thyroid hormone synthesis and protein metabolism. ${ }^{6)} \mathrm{Se}-$ rum-ferritin is an iron-binding protein that is distributed throughout the body, and serves as a biomarker of the body's iron stores. ${ }^{7)} \mathrm{Re}-$ duced serum-ferritin indicates iron deficiency, which may lead to changes in mental and cerebral mechanisms, emotion, and behavior. $^{6,8,9)}$ In contrast, chronic inflammation and oxidative stress may act as a link between elevated serum-ferritin levels, which may contribute to cellular or tissue damage. ${ }^{10)}$ A previous study revealed that increased levels of serum-ferritin were associated with major depression in patients receiving chronic hemodialysis. This suggests that systemic, lowgrade inflammation plays a role in the onset and course of the disease. $^{11)}$

Consequently, we hypothesized that serum-ferritin might be associated with mental health. However, to the best of our knowledge, few reports have demonstrated this association in large-scale human studies. $^{12,13)}$

This study aimed to investigate the association between serum-ferritin levels and mental health issues (such as short sleep duration, stress perception, depressed mood, and suicidal ideation) in the Korean elderly population, by examining large-scale, nationally representative data from the Korea Health and Nutrition Examination Survey (KNHANES) V (2010-2012), with an ultimate goal of clarifying the mechanisms and characteristics of mental health in the elderly.

\section{METHODS}

\section{Study Population}

This national, cross-sectional study was based on data from the KNHANES V survey. The KNHANES was a nationwide survey conducted by the Korean Ministry of Health and Welfare. The survey consisted of three sections: a health interview, a health examination, and a nutritional survey. The KNHANES V survey was conducted from January 2010 to December 2012 and utilized household records developed by the 2005 Population and Housing Census in Korea to select representative Korean national samples. Twenty households from each survey section were selected with a stratified, multistage probability cluster sampling system that considered geographic area, age, and sex. A total of 25,534 individuals participated in the KNHANES V survey (Figure 1). The present study included data for important analytic variables, such as serum-ferritin levels and the mental health questionnaire results from elderly individuals aged 65 years or older. We excluded participants diagnosed with anemia (hemoglobin $[\mathrm{Hb}]$ $<13 \mathrm{~g} / \mathrm{dL}$ for men, $\mathrm{Hb}<12 \mathrm{~g} / \mathrm{dL}$ for women), stroke, myocardial infarction, angina, and cancer to eliminate other factors potentially affecting serum-ferritin levels. After these adjustments, 1,802 participants were included in the statistical analysis. The Institutional Review Board of the Centers for Disease Control and Prevention in Korea approved the KNHANES examination and survey. All participants in the survey provided written informed consent (2010-02CON-21-C, 2011-02CON06-C, 2012-01EXP-01-2C).

\section{General Characteristics of the Participants}

We obtained data from the KNHANES V survey including anthropo-

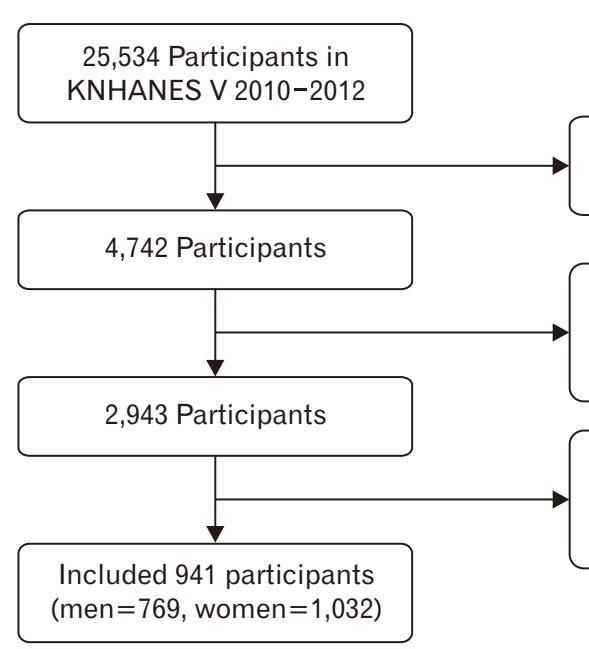

Subjects under 65 years of age $(n=20,792)$ were excluded.

Subjects who didn't have data of mental health questionnaire or serum ferritin level $(n=1,799)$ were excluded.

Subjects who diagnosed with anemia, cardiovascular disease, and cancer $(n=1,141)$ were excluded.
Figure 1. Flow diagram of the study participants. KNHANES, Korea Health and Nutrition Examination Survey. 
metric, demographic, nutritional, and medical history data. Anthropometric variables such as height and weight were measured by trained experts according to standardized protocols. Body mass index (BMI) was calculated as body weight $(\mathrm{kg})$ divided by height squared $\left(\mathrm{m}^{2}\right)$. Waist circumference was recorded midway between the lower margin of the final rib and the upper margin of the iliac crest to the nearest 0.1 $\mathrm{cm}$. Sociodemographic, lifestyle, and physical illness information was collected with a questionnaire. Marital status was divided into two groups, single or married, and education level was divided into two groups, college-graduate level or lower-than-college-graduate level. Monthly income was reported in the South Korean currency (won) and categorized into quartiles according to household income as follows: lowest, lower-middle, upper-middle, or highest. We divided the participants based on income into two groups: a low-income group (lowest or lower-middle), and a high-income group (upper-middle or highest), and also surveyed home ownership.

Regular exercise was divided into two groups: perform or do-notperform, based on whether the participants engaged in intense physical activity more than 3 days per week for at least 20 minutes, moderate physical activity more than 5 days per week for at least 30 minutes, or walking more than 5 days per week for at least 30 minutes. Regular exercise was classified according to the International Physical Activity Questionnaire. Smoking was divided into two groups based on the self-reported history of cigarette smoking, i.e., current smokers (those who were currently smoking, or had smoked more than 5 packs of cigarettes during their lifetime), and non-smokers (those who had never smoked, or had smoked less than 5 packs of cigarettes during their lifetime). Alcohol consumption was defined as having consumed at least one alcoholic drink per month in the past year. The history of comorbidity was assessed by the answers (yes or no) to questions regarding diagnosed hypertension and diabetes mellitus. Iron intake and total energy intake were assessed with the 24-hour recall method. A trained dietitian conducted the nutrition survey in the participants' homes, and used additional tools such as food models, two-dimensional food volumes, and containers to help participants recall their nutritional intake. White blood cell (WBC) counts were measured with an automated blood analyzer XE-2100 (Sysmex, Kobe, Japan), and expressed as $10^{\wedge} 3 / \mathrm{uL}$.

\section{Serum-Ferritin}

Blood samples were collected after at least 8 hours of fasting. They were processed, refrigerated immediately, and transported in cold storage to the Central Testing Institute in Seoul, Korea. Serum-ferritin levels were measured by immunofluorescence-mat ferritin (DiaSorin Inc., Stillwater, MN, USA), with a gamma counter (1470 Wizard; PerkinElmer, Turku, Finland). The participants were subdivided into quartiles based on serum-ferritin levels to determine the association between ferritin levels and mental health. The men's cut off values for quartiles were $58.52 \mathrm{ng} / \mathrm{mL}$ for the lowest quartile, $93.75 \mathrm{ng} / \mathrm{mL}$ for the second quartile, $154.04 \mathrm{ng} / \mathrm{mL}$ for the third quartile, and >154.04 ng/ $\mathrm{mL}$ for the fourth quartile. The cut-off values for women were 38.33 $\mathrm{ng} / \mathrm{mL}$ for the lowest quartile, $60.56 \mathrm{ng} / \mathrm{mL}$ for the second quartile, $93.43 \mathrm{ng} / \mathrm{mL}$ for the third quartile, and $>93.43 \mathrm{ng} / \mathrm{mL}$ for the fourth quartile.

Table 1. General characteristics of the study participants by sex

\begin{tabular}{|c|c|c|c|c|}
\hline Characteristic & Total $(\mathrm{N}=1,802)$ & Men $(\mathrm{N}=769)$ & Women $(\mathrm{N}=1,032)$ & P-value* \\
\hline Age (y) & $71.92 \pm 0.14$ & $71.50 \pm 0.30$ & $72.22 \pm 0.20$ & 0.017 \\
\hline Body mass index (kg/m²) & $24.01 \pm 0.09$ & $23.32 \pm 0.13$ & $24.51 \pm 0.19$ & $<0.001$ \\
\hline Waist circumference $(\mathrm{cm})$ & $84.54 \pm 0.27$ & $85.15 \pm 0.35$ & $84.09 \pm 0.35$ & 0.048 \\
\hline Marital status (\%) & $99.8 \pm 0.1(1,796)$ & $100 \pm 0.2(768)$ & $99.7 \pm 0.1(1,028)$ & 0.001 \\
\hline Education $^{\dagger}(\%)$ & $3.8 \pm 0.6(84)$ & $7.0 \pm 1.2(62)$ & $1.5 \pm 0.4(22)$ & $<0.001$ \\
\hline Low household income (\%) & $50.6 \pm 1.5(884)$ & $49.8 \pm 2.3(385)$ & $51.3 \pm 2.1(499)$ & 0.759 \\
\hline House ownership (\%) & $78.1 \pm 1.2(1,432)$ & $84.2 \pm 1.7(655)$ & $73.7 \pm 1.8(777)$ & $<0.001$ \\
\hline Regular exercise ${ }^{\ddagger}(\%)$ & $34.1 \pm 1.5(615)$ & $34.7 \pm 2.2(276)$ & $33.6 \pm 2.1(339)$ & 0.722 \\
\hline Current smoker (\%) & $13.0 \pm 0.9(234)$ & $26.3 \pm 1.8(198)$ & $3.5 \pm 0.7(36)$ & $<0.001$ \\
\hline Alcohol drinker (\%) & $38.0 \pm 1.4(690)$ & $68.9 \pm 2.2(482)$ & $31.1 \pm 2.2(208)$ & $<0.001$ \\
\hline Diabetes mellitus (\%) & $15.5 \pm 1.0(292)$ & $14.2 \pm 1.4(126)$ & $16.4 \pm 1.4(166)$ & 0.292 \\
\hline Hypertension (\%) & $33.4 \pm 1.9(618)$ & $30.2 \pm 2.6(30)$ & $35.6 \pm 2.6(388)$ & 0.147 \\
\hline Daily energy intake (kcal) & $1,702.49 \pm 37.65$ & $2,019.22 \pm 47.94$ & $1,474.70 \pm 21.37$ & $<0.001$ \\
\hline Daily iron intake $(\mathrm{mg})$ & $17.69 \pm 0.59$ & $18.35 \pm 1.46$ & $17.218 \pm 1.05$ & 0.437 \\
\hline White blood cell counts (10^3/uL) & $6.16 \pm 0.43$ & $6.30 \pm 0.10$ & $6.05 \pm 0.60$ & 0.009 \\
\hline Ferritin (ng/mL) & $94.29 \pm 2.28$ & $122.43 \pm 5.71$ & $74.24 \pm 2.45$ & $<0.001$ \\
\hline Short sleep duration $(\%)$ & $50.9 \pm 1.5(905)$ & $42.9 \pm 2.3(331)$ & $56.6 \pm 1.8(574)$ & $<0.001$ \\
\hline Stress perception (\%) & $21.7 \pm 1.3(386)$ & $11.6 \pm 1.4(94)$ & $28.9 \pm 1.9(292)$ & $<0.001$ \\
\hline Depressed mood (\%) & $18.2 \pm 1.2(318)$ & $11.4 \pm 1.4(87)$ & $23.2 \pm 1.7(231)$ & $<0.001$ \\
\hline Suicidal ideation (\%) & $22.5 \pm 1.3(392)$ & $15.7 \pm 1.8(114)$ & $27.4 \pm 1.8(278)$ & $<0.001$ \\
\hline
\end{tabular}

Values are presented as estimated mean \pm standard error (\%, unweighted number) for categorical variables or estimated mean \pm standard error for continuous variables. ${ }^{*}$ Obtained by Pearson's chi-square test for categorical variables or general linear model analysis for continuous variables. ${ }^{\dagger}$ Ratio of college-graduate level. ${ }^{*}$ Regular exercise includes intense physical activity more than 3 days per week for at least 20 minutes, moderate physical activity more than 5 days per week for at least 30 minutes, or walking more than 5 days per week for at least 30 minutes. ${ }^{\S}$ Sleep duration less than 7 hours. 


\section{Mental Health}

We analyzed survey data on four psychiatric symptoms. Sleep duration was divided into two groups, adequate sleep ( $\geq 7$ hours) and inadequate sleep ( $<7$ hours). ${ }^{14)}$ Stress perception was assessed by self-reports with the following question: "How stressed do you feel during your usual life activities?" Based on the report, the participants were classified into two categories, a high-stress group and a low-stress group. Depressed mood included feeling sadness or despair continuously for $>2$ weeks during the past year, to a degree sufficient to interfere with daily life. The participants were divided into two groups, depression and non-depression. We also asked participants if they had experienced suicidal ideation during the past year.

\section{Statistical Analysis}

We obtained data by complex sampling and considered strata, clusters, and weights. All analyses were performed with the sample weights from the KNHANES V survey. The clinical characteristics of men and women were compared using the Pearson chi-square test for categorical variables, and a generalized linear model for continuous variables. Data for continuous variables are expressed as mean \pm standard error, and data for categorical variables are expressed as mean \pm standard error (\%, unweighted number). We performed multiple logistic regression analyses to calculate the odds ratios and $95 \%$ confidence intervals, for the association between serum-ferritin levels and mental health. Age, BMI, waist circumference, education level, income level, housing status, physical activity, smoking status, alcohol con- sumption, history of diabetes mellitus or hypertension, energy intake, iron intake, and WBC counts were adjusted. All analyses were performed using IBM SPSS for Windows ver. 21.0 (IBM Corp., Armonk, NY, USA). A P for trend $<0.05$ was considered statistically significant.

\section{RESULTS}

\section{General Characteristics of the Participants}

Table 1 shows the general characteristics of the participants. The study included 769 men and 1,032 women. The mean serum-ferritin level in men was significantly higher than that in women $(122.43 \pm 5.71$ versus $74.24 \pm 2.45 \mathrm{ng} / \mathrm{mL}$, respectively; $\mathrm{P}<0.001)$. Overall, women were older, and had a higher BMI than men. Women had a lower education level and housing status, and were less likely to be smokers or consume alcohol than men. Psychiatric symptoms showed a higher rate in women (short sleep duration [ $<7$ hours]: $42.9 \% \pm 2.3 \%$ versus $56.6 \% \pm 1.8 \%$; stress perception: $11.6 \% \pm 1.4 \%$ versus $28.9 \% \pm 1.9 \%$; depressed mood: $11.4 \% \pm 1.4 \%$ versus $23.2 \% \pm 1.7 \%$; and suicidal ideation: $15.7 \% \pm 1.8 \%$ versus $27.4 \% \pm 1.8 \%)$.

\section{General Characteristics of the Participants according to the Quartiles of Serum-Ferritin Levels}

The characteristics of the study participants according to the serumferritin quartiles of men and women are shown in Table 2 and Table 3, respectively. Men with low serum-ferritin levels had low household incomes $(\mathrm{P}=0.011)$. There were also significant differences in alcohol

Table 2. General characteristics of men, according to the quartiles of serum-ferritin levels

\begin{tabular}{|c|c|c|c|c|c|}
\hline Characteristic & Q1 $(N=194)$ & Q2 (N=189) & Q3 $(N=195)$ & Q4 (N=191) & $P$-value* \\
\hline Serum-ferritin (ng/mL) & $38.88 \pm 15.48$ & $75.78 \pm 15.63$ & $120.21 \pm 15.38$ & $267.29 \pm 15.61$ & \\
\hline Age $(y)$ & $71.84 \pm 0.57$ & $70.94 \pm 0.57$ & $71.61 \pm 0.57$ & $71.63 \pm 0.42$ & 0.459 \\
\hline Body mass index (kg/m²) & $22.83 \pm 0.38$ & $23.58 \pm 0.36$ & $23.39 \pm 0.40$ & $23.55 \pm 0.27$ & 0.131 \\
\hline Waist circumference (cm) & $83.93 \pm 1.06$ & $85.75 \pm 0.99$ & $84.89 \pm 1.04$ & $86.25 \pm 0.72$ & 0.141 \\
\hline Marital status (\%) & $99.0 \pm 0.6(193)$ & $100 \pm 0.0(189)$ & $100 \pm 0.0(195)$ & $100 \pm 0.0(191)$ & 0.911 \\
\hline Education ${ }^{\dagger}(\%)$ & $6.7 \pm 2.1(14)$ & $6.1 \pm 2.3(14)$ & $5.8 \pm 1.8(16)$ & $9.4 \pm 2.8(18)$ & 0.644 \\
\hline Low household income (\%) & $46.1 \pm 4.1(99)$ & $50.3 \pm 4.4(88)$ & $49.9 \pm 4.3(103)$ & $53.4 \pm 4.7(95)$ & 0.011 \\
\hline House ownership (\%) & $82.8 \pm 3.3(163)$ & $82.8 \pm 4.0(161)$ & $88.2 \pm 2.6(169)$ & $83.2 \pm 3.7(162)$ & 0.610 \\
\hline Regular exercise ${ }^{\ddagger}(\%)$ & $32.6 \pm 4.0(61)$ & $42.2 \pm 4.3(85)$ & $32.5 \pm 4.4(68)$ & $31.4 \pm 4.3(62)$ & 0.248 \\
\hline Current smoker (\%) & $25.4 \pm 3.9(43)$ & $25.0 \pm 3.6(50)$ & $26.8 \pm 3.7(48)$ & $28.2 \pm 4.2(57)$ & 0.936 \\
\hline Alcohol drinker (\%) & $55.2 \pm 5.0(103)$ & $68.8 \pm 4.0(126)$ & $56.7 \pm 4.2(115)$ & $70.1 \pm 3.7(138)$ & 0.024 \\
\hline Diabetes mellitus (\%) & $12.3 \pm 2.4(27)$ & $17.2 \pm 3.3(41)$ & $12.4 \pm 2.5(30)$ & $15.3 \pm 2.9(36)$ & 0.522 \\
\hline Hypertension (\%) & $17.5 \pm 3.2(45)$ & $36.6 \pm 4.6(66)$ & $32.5 \pm 5.2(62)$ & $35.7 \pm 4.5(57)$ & 0.003 \\
\hline Daily energy intake (kcal) & $1,984.18 \pm 101.43$ & $2,131.31 \pm 98.64$ & $1,959.70 \pm 124.03$ & $2,004.17 \pm 74.52$ & 0.365 \\
\hline Daily iron intake (mg) & $16.75 \pm 2.03$ & $20.66 \pm 2.65$ & $18.42 \pm 2.33$ & $17.65 \pm 1.63$ & 0.499 \\
\hline White blood cell counts (10^3/uL) & $6.18 \pm 0.19$ & $6.51 \pm 0.22$ & $6.22 \pm 0.18$ & $6.31 \pm 0.14$ & 0.431 \\
\hline Short sleep duration ${ }^{\S}(\%)$ & $38.7 \pm 4.2(85)$ & $44.0 \pm 4.1(83)$ & $43.6 \pm 4.3(80)$ & $45.7 \pm 4.4(83)$ & 0.646 \\
\hline Stress perception (\%) & $11.4 \pm 2.8(24)$ & $14.6 \pm 3.0(25)$ & $8.7 \pm 2.1(22)$ & $11.8 \pm 2.9(23)$ & 0.491 \\
\hline Depressed mood (\%) & $12.7 \pm 3.2(21)$ & $12.6 \pm 3.1(20)$ & $12.0 \pm 2.7(26)$ & $8.0 \pm 1.8(20)$ & 0.571 \\
\hline Suicidal ideation (\%) & $17.3 \pm 3.2(30)$ & $21.0 \pm 4.5(31)$ & $11.2 \pm 2.7(23)$ & $13.3 \pm 2.8(30)$ & 0.148 \\
\hline
\end{tabular}

Values are presented as estimated mean \pm standard error (\%, unweighted number) for categorical variables or estimated mean \pm standard error for continuous variables. Q, quartile.

${ }^{*}$ Obtained by Pearson's chi-square test for categorical variables or general linear model analysis for continuous variables. ${ }^{\dagger}$ Ratio of college-graduate level. ${ }^{\ddagger}$ Regular exercise includes intense physical activity more than 3 days per week for at least 20 minutes, moderate physical activity more than 5 days per week for at least 30 minutes, or walking more than 5 days per week for at least 30 minutes. ${ }^{\S}$ Sleep duration less than 7 hours. 
Table 3. General characteristics of women, according to the quartiles of serum-ferritin levels

\begin{tabular}{|c|c|c|c|c|c|}
\hline Characteristic & Q1 (N=265) & Q2 $(\mathrm{N}=256)$ & Q3 (N=252) & Q4 (N=259) & P-value* \\
\hline Serum-ferritin (ng/mL) & $25.33 \pm 5.70$ & $48.75 \pm 5.68$ & $76.65 \pm 5.62$ & $146.53 \pm 5.62$ & \\
\hline $\operatorname{Age}(y)$ & $72.15 \pm 0.51$ & $71.69 \pm 0.53$ & $72.34 \pm 0.63$ & $72.72 \pm 0.40$ & 0.264 \\
\hline Body mass index (kg/m²) & $24.49 \pm 0.33$ & $24.20 \pm 0.35$ & $24.40 \pm 0.35$ & $24.94 \pm 0.27$ & 0.194 \\
\hline Waist circumference (cm) & $84.01 \pm 0.90$ & $82.95 \pm 0.97$ & $84.00 \pm 0.94$ & $85.40 \pm 0.73$ & 0.093 \\
\hline Marital status (\%) & $99.9 \pm 0.2(264)$ & $99.7 \pm 0.3(255)$ & $99.8 \pm 0.2(251)$ & $99.6 \pm 0.4(258)$ & 0.906 \\
\hline Education ${ }^{\dagger}(\%)$ & $0.6 \pm 0.3(3)$ & $2.1 \pm 1.1(5)$ & $1.5 \pm 0.8(5)$ & $2.0 \pm 0.8(9)$ & 0.420 \\
\hline Low household income (\%) & $54.3 \pm 3.7(135)$ & $52.8 \pm 4.1(128)$ & $50.9 \pm 4.3(118)$ & $47.0 \pm 3.9(118)$ & 0.796 \\
\hline House ownership (\%) & $72.6 \pm 3.2(193)$ & $72.8 \pm 3.6(195)$ & $76.5 \pm 3.3(192)$ & $72.9 \pm 3.2(197)$ & 0.811 \\
\hline Regular exercise ${ }^{\ddagger}(\%)$ & $31.5 \pm 3.7(85)$ & $37.7 \pm 4.0(92)$ & $34.4 \pm 3.8(86)$ & $30.9 \pm 3.4(76)$ & 0.518 \\
\hline Current smoker (\%) & $4.0 \pm 1.3(11)$ & $2.6 \pm 1.3(7)$ & $3.2 \pm 1.2(7)$ & $4.2 \pm 1.5(11)$ & 0.827 \\
\hline Alcohol drinker (\%) & $19.9 \pm 3.2(50)$ & $17.7 \pm 2.8(47)$ & $23.6 \pm 3.3(61)$ & $20.4 \pm 3.2(50)$ & 0.634 \\
\hline Diabetes mellitus (\%) & $15.8 \pm 2.5(43)$ & $14.9 \pm 2.5(41)$ & $17.1 \pm 3.1(38)$ & $17.7 \pm 2.8(44)$ & 0.878 \\
\hline Hypertension (\%) & $41.2 \pm 4.2(111)$ & $33.6 \pm 3.8(96)$ & $30.4 \pm 3.8(82)$ & $36.9 \pm 4.4(99)$ & 0.193 \\
\hline Daily energy intake (kcal) & $1,471.63 \pm 55.16$ & $1,487.77 \pm 56.52$ & $1,474.15 \pm 61.92$ & $1,465.15 \pm 43.98$ & 0.980 \\
\hline Daily iron intake (mg) & $15.93 \pm 2.64$ & $18.47 \pm 2.70$ & $15.24 \pm 2.75$ & $19.06 \pm 2.18$ & 0.423 \\
\hline White blood cell counts (10^3/uL) & $6.00 \pm 0.17$ & $6.00 \pm 0.17$ & $5.92 \pm 0.19$ & $6.28 \pm 0.12$ & 0.202 \\
\hline Short sleep duration ${ }^{\S}(\%)$ & $51.2 \pm 3.5(142)$ & $57.0 \pm 3.7(147)$ & $63.2 \pm 3.9(144)$ & $55.4 \pm 3.8(141)$ & 0.164 \\
\hline Stress perception (\%) & $35.5 \pm 3.5(91)$ & $28.2 \pm 3.4(69)$ & $24.4 \pm 3.3(66)$ & $27.4 \pm 3.7(66)$ & 0.126 \\
\hline Depressed mood (\%) & $28.0 \pm 3.4(71)$ & $19.5 \pm 2.9(51)$ & $24.3 \pm 3.6(53)$ & $20.8 \pm 3.1(56)$ & 0.219 \\
\hline Suicidal ideation (\%) & $30.4 \pm 3.4(81)$ & $24.2 \pm 3.4(64)$ & $24.8 \pm 3.6(58)$ & $30.1 \pm 3.6(75)$ & 0.421 \\
\hline
\end{tabular}

Values are presented as estimated mean \pm standard error (\%, unweighted number) for categorical variables or estimated mean \pm standard error for continuous variables. $Q$, quartile.

*Obtained by Pearson's chi-square test for categorical variables or general linear model analysis for continuous variables. 'Ratio of college-graduate level. ${ }^{\star}$ Regular exercise includes intense physical activity more than 3 days per week for at least 20 minutes, moderate physical activity more than 5 days per week for at least 30 minutes, or walking more than 5 days per week for at least 30 minutes. ${ }^{\circledR}$ Sleep duration less than 7 hours.

consumption $(\mathrm{P}=0.024)$, and prevalence of hypertension $(\mathrm{P}=0.003)$, according to the men's serum-ferritin quartiles. In women, there was no significant difference in general characteristics according to the serum-ferritin quartiles.

\section{Association between Serum-Ferritin Levels and Mental Health}

Tables 4 and 5 present the ORs (95\% confidence intervals) of mental health in men and women, respectively, according to the serum-ferritin quartiles. Model 1 was adjusted for age, BMI, and waist circumference. Model 2 was adjusted for age, BMI, waist circumference, education level, income level, housing status, physical activity, smoking status, alcohol consumption, history of diabetes mellitus or hypertension, energy intake, iron intake, and WBC counts. In the multiple logistic regression analysis, serum-ferritin level was significantly associated with suicidal ideation in the fully adjusted model in men (Q1:1 [reference], Q2:1.132 [95\%CI, 0.569-2.252], Q3:0453 [95\%CI, 0.217-0.945], Q4:0.608 [95\%CI, 0.295-1.253]; P for trend=0.039). In women, no trend was observed; however, compared to the lowest quartile, short sleep duration ( $<7$ hours) and stress perception were significantly decreased in the third quartile, and the odds ratios were 0.601 (95\% CI, 0.406-0.888) and 0.606 (95\% CI, 0.386-0.952), respectively.

\section{DISCUSSION}

This study examined the association between serum-ferritin levels and mental health in a nationally representative sample of older Koreans. To the best of our knowledge, this is the first reported study to explore the link between serum-ferritin and mental health.

We found that men with lower serum-ferritin levels exhibited a trend for higher risk of suicidal ideation, and compared to the lowest quartile, suicidal ideation was significantly decreased in the third quartile. High-impulsivity has been linked with low-iron status in some observational and experimental studies. Moscicki ${ }^{15)}$ reported that low-iron status may be associated with suicide via the pathway of increased impulsivity. A sufficient amount of iron is particularly important for the synthesis of serotonin, a neurotransmitter that plays a significant role in mood disorders and impulsivity. ${ }^{13,16)}$ Studies on the serotonergic system have found that serotonin was reduced in the ventral prefrontal cortex region, resulting in impaired inhibition and a greater propensity to act on powerful feelings such as suicidal or aggressive feelings. ${ }^{13)}$ However, in the present study, depressed mood, a considerable cause for suicide, was not associated with ferritin level. This result may be related to the characteristics of elderly depression. In the elderly, depressed mood may be less prominent than other symptoms, such as loss of interest and appetite, sleeplessness, and anergia. $^{17)}$

Our study also found that moderately elevated serum-ferritin (Q3) levels were associated with a decreased risk of short sleep duration in women. This association was also independent of several confounders. The relationship between sleep duration and serum-ferritin is widely known, and low ferritin level has been associated with restless 
Table 4. OR and $95 \% \mathrm{Cl}$ of psychiatric symptoms according to the quartiles of serum-ferritin in men

\begin{tabular}{|c|c|c|c|c|c|c|c|}
\hline \multirow{2}{*}{ Variable } & \multirow{2}{*}{$Q$} & \multicolumn{2}{|c|}{ Unadjusted } & \multicolumn{2}{|l|}{ Model 1} & \multicolumn{2}{|l|}{ Model 2} \\
\hline & & OR (95\% Cl) & P-value & $\mathrm{OR}(95 \% \mathrm{Cl})$ & P-value & OR $(95 \% \mathrm{Cl})$ & $P$ for trend ${ }^{*}$ \\
\hline \multirow[t]{4}{*}{ Short sleep duration } & Q1 & 1 (Reference) & \multirow[t]{4}{*}{0.280} & 1 (Reference) & \multirow[t]{4}{*}{0.307} & 1 (Reference) & \multirow[t]{4}{*}{0.468} \\
\hline & Q2 & $0.801(0.505-1.269)$ & & $0.818(0.513-1.303)$ & & $0.842(0.521-1.359)$ & \\
\hline & Q3 & $0.815(0.494-1.344)$ & & $0.844(0.512-1.392)$ & & $0.859(0.512-1.441)$ & \\
\hline & Q4 & $0.748(0.461-1.216)$ & & $0.752(0.460-1.227)$ & & $0.810(0.490-1.339)$ & \\
\hline \multirow[t]{4}{*}{ Stress perception } & Q1 & 1 (Reference) & \multirow[t]{4}{*}{0.728} & 1 (Reference) & \multirow[t]{4}{*}{0.803} & 1 (Reference) & \multirow[t]{4}{*}{0.827} \\
\hline & Q2 & $1.330(0.656-2.696)$ & & $0.731(0.361-1.483)$ & & $1.349(0.664-2.740)$ & \\
\hline & Q3 & $0.746(0.362-1.535)$ & & $1.302(0.636-2.666)$ & & $0.791(0.380-1.614)$ & \\
\hline & Q4 & $1.048(0.482-2.277)$ & & $0.917(0.426-1.972)$ & & $1.104(0.509-2.396)$ & \\
\hline \multirow[t]{4}{*}{ Depressed mood } & Q1 & 1 (Reference) & \multirow[t]{4}{*}{0.230} & 1 (Reference) & \multirow[t]{4}{*}{0.215} & 1 (Reference) & \multirow[t]{4}{*}{0.174} \\
\hline & Q2 & $0.996(0.448-2.213)$ & & $0.996(0.448-2.214)$ & & $0.818(0.355-1.884)$ & \\
\hline & Q3 & $0.940(0.443-1.996)$ & & $0.944(0.448-1.991)$ & & $0.855(0.381-1.920)$ & \\
\hline & Q4 & $0.599(0.283-1.268)$ & & $0.587(0.276-1.252)$ & & $0.540(0.241-1.213)$ & \\
\hline \multirow[t]{4}{*}{ Suicidal ideation } & Q1 & 1 (Reference) & \multirow[t]{4}{*}{0.125} & 1 (Reference) & \multirow[t]{4}{*}{0.141} & 1 (Reference) & \multirow[t]{4}{*}{0.039} \\
\hline & Q2 & $1.275(0.674-0.414)$ & & $1.365(0.715-2.607)$ & & $1.132(0.569-2.252)$ & \\
\hline & Q3 & $0.604(0.300-1.215)$ & & $0.602(0.300-1.210)$ & & $0.453(0.217-0.945)$ & \\
\hline & Q4 & $0.732(0.378-1.416)$ & & $0.762(0.387-1.500)$ & & $0.608(0.295-1.253)$ & \\
\hline
\end{tabular}

Values are presented as OR $(95 \% \mathrm{Cl})$. ORs and $95 \%$ Cls were estimated by multiple logistic regression analyses. Model 1: adjusted for age, body mass index, and waist circumference; Model 2: adjusted for age, body mass index, and waist circumference, education level, income level, house ownership, physical activity, smoking status, alcohol consumption, history of diabetes mellitus or hypertension, energy intake, iron intake, and white blood cell counts.

$\mathrm{OR}$, odds ratio; $\mathrm{Cl}$, confidence interval; $\mathrm{Q}$, quartile.

*Assessed by generalized linear model in which ferritin quartile was put as a continuous variable.

Table 5. OR and 95\% Cl of psychiatric symptoms according to the quartiles of serum-ferritin in women

\begin{tabular}{|c|c|c|c|c|c|c|c|}
\hline \multirow{2}{*}{ Variable } & \multirow{2}{*}{$Q$} & \multicolumn{2}{|c|}{ Unadjusted } & \multicolumn{2}{|l|}{ Model 1} & \multicolumn{2}{|l|}{ Model 2} \\
\hline & & $\mathrm{OR}(95 \% \mathrm{Cl})$ & P-value & $\mathrm{OR}(95 \% \mathrm{Cl})$ & P-value & OR $(95 \%$ Cl) & P for trend ${ }^{\star}$ \\
\hline \multirow[t]{4}{*}{ Short sleep duration } & Q1 & 1 (Reference) & \multirow[t]{4}{*}{0.248} & 1 (Reference) & \multirow[t]{4}{*}{0.234} & 1 (Reference) & \multirow[t]{4}{*}{0.307} \\
\hline & Q2 & $0.790(0.525-1.188)$ & & $0.787(0.521-1.190)$ & & $0.786(0.514-1.203)$ & \\
\hline & Q3 & $0.611(0.418-0.893)$ & & $0.606(0.414-0.887)$ & & $0.601(0.406-0.888)$ & \\
\hline & Q4 & $0.845(0.560-1.277)$ & & $0.841(0.556-1.272)$ & & $0.864(0.564-1.322)$ & \\
\hline \multirow[t]{4}{*}{ Stress perception } & Q1 & 1 (Reference) & \multirow[t]{4}{*}{0.060} & 1 (Reference) & \multirow[t]{4}{*}{0.063} & 1 (Reference) & \multirow[t]{4}{*}{0.113} \\
\hline & Q2 & $0.713(0.460-1.105)$ & & $0.704(0.453-1.093)$ & & $0.627(0.401-0.980)$ & \\
\hline & Q3 & $0.586(0.374-0.917)$ & & $0.585(0.374-0.915)$ & & $0.606(0.386-0.952)$ & \\
\hline & Q4 & $0.684(0.443-1.058)$ & & $0.689(0.446-1.065)$ & & $0.690(0.434-1.097)$ & \\
\hline \multirow[t]{4}{*}{ Depressed mood } & Q1 & 1 (Reference) & \multirow[t]{4}{*}{0.242} & 1 (Reference) & \multirow[t]{4}{*}{0.171} & 1 (Reference) & \multirow[t]{4}{*}{0.153} \\
\hline & Q2 & $0.622(0.382-1.010)$ & & $0.524(0.318-0.864)$ & & $0.515(0.327-0.941)$ & \\
\hline & Q3 & $0.826(0.497-1.370)$ & & $0.818(0.485-1.381)$ & & $0.790(0.452-1.382)$ & \\
\hline & Q4 & $0.673(0.408-1.109)$ & & $0.552(0.323-0.944)$ & & $0.486(0.275-0.852)$ & \\
\hline \multirow[t]{4}{*}{ Suicidal ideation } & Q1 & 1 (Reference) & \multirow[t]{4}{*}{0.984} & 1 (Reference) & \multirow[t]{4}{*}{0.889} & 1 (Reference) & \multirow[t]{4}{*}{0.872} \\
\hline & Q2 & $0.729(0.444-1.198)$ & & $0.736(0.449-1.209)$ & & $0.667(0.388-1.145)$ & \\
\hline & Q3 & $0.755(0.491-1.159)$ & & $0.751(0.490-1.153)$ & & $0.780(0.497-1.225)$ & \\
\hline & Q4 & 0.985(0.627-1.546) & & $0.963(0.611-1.516)$ & & $0.940(0.572-1.544)$ & \\
\hline
\end{tabular}

Values are presented as OR $(95 \% \mathrm{Cl})$. ORs and $95 \%$ Cls were estimated by multiple logistic regression analyses. Model 1: adjusted for age, body mass index, and waist circumference; Model 2: adjusted for age, body mass index, and waist circumference, education level, income level, house ownership, physical activity, smoking status, alcohol consumption, history of diabetes mellitus or hypertension, energy intake, iron intake, and white blood cell counts.

$\mathrm{OR}$, odds ratio; $\mathrm{Cl}$, confidence interval; $\mathrm{Q}$, quartile.

*Assessed by generalized linear model in which ferritin quartile was put as a continuous variable.

leg syndrome (which disturbs sleep), and in a previous randomized blind study of restless leg syndrome subjects, the symptoms were relieved after oral iron supplementation. ${ }^{18)}$ However, although an association with ferritin and dopamine was shown, the ultimate mechanism for restless leg syndrome was not elucidated.

Stress perception was also decreased in older women with moder- ately elevated serum-ferritin levels. Several previous studies have explored the relationship between serum- ferritin and mental health, (mainly depression), with conflicting results. ${ }^{19,20)}$ However, few studies have investigated the effect of iron status on stress, and the precise mechanism by which lower serum-ferritin levels might cause stress remains unclear. ${ }^{12)} \mathrm{A}$ possible role of iron in brain function and emo- 
tional behavior is suggested by the association of mental health with decreased serum-ferritin levels. ${ }^{9,21,22)}$ Poor iron status, particularly advanced iron deficiency, alters myelination, and neurotransmitter metabolism and function in brain regions such as the hippocampus, striatum, and cortex. ${ }^{23)}$ In particular, a sufficient amount of iron is needed for the synthesis of dopamine, a neurotransmitter that plays an important role in mood disorders. ${ }^{24)}$

Additionally, the observed relationship between decreased serumferritin concentration and mental health differed in men and women in this study. The inconsistencies may simply be due to the differences in total serum-ferritin levels in men and women, since lower serumferritin concentrations were more common in women. Ikeda et al. ${ }^{25}$ found that estrogen regulates hepcidin, a liver-derived iron regulatory protein, which plays a crucial role in iron metabolism. A recent animal study showed that the serotonin transporter decreased in the frontal cortex, but increased in the nucleus accumbens in females, if an iron deficiency was present. In males, an increase in the serotonin and dopamine transporters was observed in the nucleus accumbens with iron deficiency. ${ }^{26)}$

However, the association of mental health and ferritin in males and females was observed in the moderate, but not high ferritin level subjects in our study. Serum-ferritin is an iron storage protein but also an inflammatory marker that may be released from damaged cells, and could be a marker of cellular damage. ${ }^{27)}$ Therefore, increased ferritin may be caused by inflammation (and not iron overload) in subjects with a high ferritin level. Consequently, the mental health and ferritin association may be complex. For example, in another study, high ferritin levels were associated with liver or renal disease, and malignancy. ${ }^{28)}$ In addition, in our study, daily iron intake was not significantly different in each group; hence, the cause for the high ferritin levels was unclear.

The principal strengths of our study were the large-scale, nationally representative sample of older Koreans, and the investigation of the association between serum-ferritin levels and mental health after stratification by sex. We also adjusted for potentially confounding factors that could influence the results. Mental health is associated with many socioeconomic and lifestyle characteristics. In addition, serumferritin levels are related to patient disease history, such as hypertension and diabetes mellitus. Also, inflammatory markers, such as WBC counts, may be additional confounding factors. ${ }^{29)}$

However, this study had some limitations. First, this was a cross-sectional study, and thus we could not identify a direct causative relationship between serum-ferritin levels and mental health. Hence, further prospective studies should be conducted to establish a causal relationship between serum-ferritin levels and mental health. Second, mental health was evaluated by self-reported questionnaires, and such research instruments may not constitute a sufficient assessment. Third, cognitive function was not included in our report because of lack of data. Cognitive function is an important reflection of mental health in the elderly; therefore, further study is needed.

In conclusion, low serum-ferritin levels were associated with an in- creased suicidal ideation in Korean elderly men, and increased risk of short sleep duration and stress perception in Korean elderly women.

\section{CONFLICT OF INTEREST}

No potential conflict of interest relevant to this article was reported.

\section{ORCID}

Kyung Mi Kim: https://orcid.org/0000-0003-1867-8082

Hye Rim Hwang: https://orcid.org/0000-0001-7658-3749

Yun Jin Kim: https://orcid.org/0000-0002-0204-3253

Jeong Gyu Lee: https://orcid.org/0000-0001-7160-0714

Yu Hyeon Yi: https://orcid.org/0000-0002-1786-2737

Young Jin Tak: https://orcid.org/0000-0002-4645-5866

Seung Hun Lee: https://orcid.org/0000-0002-0976-8708

Sung In Chung: https://orcid.org/0000-0003-2289-0596

\section{REFERENCES}

1. Tanaka H, Taira K, Arakawa M, Urasaki C, Yamamoto Y, Okuma H, et al. Short naps and exercise improve sleep quality and mental health in the elderly. Psychiatry Clin Neurosci 2002;56:233-4.

2. Shin HC. Measuring stress with questionnaires. J Korean Med Assoc 2013;56:485-95.

3. Cho M, Sung S, Shin S, Kim J, Jeon S, Kim M. The epidemiological survey of mental disorders in Korea. Sejong: Ministry of Health and Welfare; 2011.

4. Organization for Economic Cooperation and Development. Health status: suicide: trends in suicide, selected OECD countries, 1990-2013. In: Organization for Economic Cooperation and Development. Health at a glance 2015. Paris: Organization for Economic Cooperation and Development; 2015. p. 57.

5. Looker AC, Dallman PR, Carroll MD, Gunter EW, Johnson CL. Prevalence of iron deficiency in the United States. JAMA 1997;277:973-6.

6. Beard J. Iron deficiency alters brain development and functioning. J Nutr 2003;133(5 Suppl 1):1468S-72S.

7. Cook JD, Flowers $\mathrm{CH}$, Skikne BS. The quantitative assessment of body iron. Blood 2003;101:3359-64.

8. Lozoff B. Behavioral alterations in iron deficiency. Adv Pediatr 1988;35:331-59.

9. Kim J, Wessling-Resnick M. Iron and mechanisms of emotional behavior. J Nutr Biochem 2014;25:1101-7.

10. Furukawa S, Fujita T, Shimabukuro M, Iwaki M, Yamada Y, Nakajima Y, et al. Increased oxidative stress in obesity and its impact on metabolic syndrome. J Clin Invest 2004;114:1752-61.

11. Huang TL, Lee CT. Low serum albumin and high ferritin levels in chronic hemodialysis patients with major depression. Psychiatry Res 2007;152:277-80.

12. Chen J, Shen H, Chen C, Wang W, Yu S, Zhao M, et al. The effect of psychological stress on iron absorption in rats. BMC Gastroenterol 2009;9:83.

13. Li Y, Dai Q, Torres ME, Zhang J. Gender-specific association between iron status and the history of attempted suicide: implications for gen- 
der paradox of suicide behaviors. Prog Neuropsychopharmacol Biol Psychiatry 2007;31:1429-35.

14. Hirshkowitz M, Whiton K, Albert SM, Alessi C, Bruni O, DonCarlos L, et al. National Sleep Foundation's sleep time duration recommendations: methodology and results summary. Sleep Health 2015;1:40-3.

15. Moscicki EK. Gender differences in completed and attempted suicides. Ann Epidemiol 1994;4:152-8.

16. Yi S, Nanri A, Poudel-Tandukar K, Nonaka D, Matsushita Y, Hori A, et al. Association between serum ferritin concentrations and depressive symptoms in Japanese municipal employees. Psychiatry Res 2011; 189:368-72.

17. Lebowitz BD, Pearson JL, Schneider LS, Reynolds CF 3rd, Alexopoulos GS, Bruce ML, et al. Diagnosis and treatment of depression in late life: consensus statement update. JAMA 1997;278:1186-90.

18. Wang J, O'Reilly B, Venkataraman R, Mysliwiec V, Mysliwiec A. Efficacy of oral iron in patients with restless legs syndrome and a low-normal ferritin: a randomized, double-blind, placebo-controlled study. Sleep Med 2009;10:973-5.

19. Baune BT, Eckardstein Av, Berger K. Lack of association between iron metabolism and depressive mood in an elderly general population. Int Psychogeriatr 2006;18:437-44.

20. Maes M, van de Vyvere J, Vandoolaeghe E, Bril T, Demedts P, Wauters A, et al. Alterations in iron metabolism and the erythron in major depression: further evidence for a chronic inflammatory process. J Affect Disord 1996;40:23-33.

21. Vahdat Shariatpanaahi M, Vahdat Shariatpanaahi Z, Moshtaaghi M, Shahbaazi SH, Abadi A. The relationship between depression and se- rum ferritin level. Eur J Clin Nutr 2007;61:532-5.

22. Baune BT, Neuhauser H, Ellert U, Berger K. The role of the inflammatory markers ferritin, transferrin and fibrinogen in the relationship between major depression and cardiovascular disorders: the German Health Interview and Examination Survey. Acta Psychiatr Scand 2010;121:135-42.

23. Lozoff B, Beard J, Connor J, Barbara F, Georgieff M, Schallert T. Longlasting neural and behavioral effects of iron deficiency in infancy. Nutr Rev 2006;64(5 Pt 2):S34-43.

24. Insel P, Turner RE, Ross D. Nutrition. 2nd ed. Sudbury (MA): Jones and Bartlett Publishers; 2002.

25. Ikeda Y, Tajima S, Izawa-Ishizawa Y, Kihira Y, Ishizawa K, Tomita S, et al. Estrogen regulates hepcidin expression via GPR30-BMP6-dependent signaling in hepatocytes. PLoS One 2012;7:e40465.

26. Morse AC, Beard JL, Azar MR, Jones BC. Sex and genetics are important cofactors in assessing the impact of iron deficiency on the developing mouse brain. Nutr Neurosci 1999;2:323-35.

27. Kell DB, Pretorius E. Serum ferritin is an important inflammatory disease marker, as it is mainly a leakage product from damaged cells. Metallomics 2014;6:748-73.

28. Cullis JO, Fitzsimons EJ, Griffiths WJ, Tsochatzis E, Thomas DW; British Society for Haematology. Investigation and management of a raised serum ferritin. Br J Haematol 2018;181:331-40.

29. Miller AH, Raison CL. The role of inflammation in depression: from evolutionary imperative to modern treatment target. Nat Rev Immunol 2016;16:22-34. 